\title{
Effects of Sizes and Conformations of Fish-Scale Collagen Peptides on Facial Skin Qualities and Transdermal Penetration Efficiency
}

\author{
Huey-Jine Chai, ${ }^{1,2}$ Jing-Hua Li, ${ }^{2}$ Han-Ning Huang, ${ }^{1}$ Tsung-Lin Li, ${ }^{3}$ Yi-Lin Chan, ${ }^{4}$ \\ Chyuan-Yuan Shiau, ${ }^{1}$ and Chang-Jer $\mathrm{Wu}^{1}$ \\ ${ }^{1}$ Department of Food Science, National Taiwan Ocean University, Keelung 20224, Taiwan \\ ${ }^{2}$ Seafood Technology Division, Fisheries Research Institute, Council of Agriculture, Keelung 20246, Taiwan \\ ${ }^{3}$ Genomics Research Center, Academia Sinica, Taipei 11529, Taiwan \\ ${ }^{4}$ Institute of Biomedical Sciences, Academia Sinica, Taipei 11529, Taiwan
}

Correspondence should be addressed to Chang-Jer Wu, wuchangjer@yahoo.com.tw

Received 25 February 2010; Accepted 3 April 2010

Academic Editor: Rumiana Koynova

Copyright (C) 2010 Huey-Jine Chai et al. This is an open access article distributed under the Creative Commons Attribution License, which permits unrestricted use, distribution, and reproduction in any medium, provided the original work is properly cited.

Fish-scale collagen peptides (FSCPs) were prepared using a given combination of proteases to hydrolyze tilapia (Oreochromis sp.) scales. FSCPs were determined to stimulate fibroblast cells proliferation and procollagen synthesis in a time- and dose-dependent manner. The transdermal penetration capabilities of the fractionationed FSCPs were evaluated using the Franz-type diffusion cell model. The heavier FSCPs, 3500 and $4500 \mathrm{Da}$, showed higher cumulative penetration capability as opposed to the lighter FSCPs, 2000 and $1300 \mathrm{Da}$. In addition, the heavier seemed to preserve favorable coiled structures comparing to the lighter that presents mainly as linear under confocal scanning laser microscopy. FSCPs, particularly the heavier, were concluded to efficiently penetrate stratum corneum to epidermis and dermis, activate fibroblasts, and accelerate collagen synthesis. The heavier outweighs the lighter in transdermal penetration likely as a result of preserving the given desired structure feature.

\section{Introduction}

Collagens are the major structural element of connective tissues in vertebrate, comprising $30 \%$ or so of total protein. They also exist in the interstitial tissues of virtually all parenchymal organs, wherein they stabilize organs and keep them in good shapes [1]. To date, over 20 types of collagens have been identified, and they were otherwise divided into three groups, fibrous collagen, fibril associated collagen, and basement membrane collagen [1-3]. Skin, the largest organ in human body protects the body from varied external insults. It is composed of three layers, namely the epidermis, the dermis, and the subcutaneous layer. Each layer provides some extents of physical strength and flexibility in concert with other physiological functions [4]. The dermis contains considerable amounts of extracellular matrix (ECM), such as collagens and glycosaminoglycans (GAGs) produced mainly by fibroblasts. Collagen type I [5] known to maintain the integral of the dermis is the most abundant collagen in human body. Aging skin is admittedly related to the reduction of collagen type I [6].

Collagen has been widely processed as products in food, cosmetic, biomedical, and pharmaceutical industries. Oral consumption of collagen peptides may provide some beneficial effects for the body. For examples, collagen peptides as a food supplement may improve low bone mineral density in people in malnutrition and people suffering degenerative joint diseases [7-9]. Reports also indicated that consumption of collagen can thicken hairs [10], improve nail disorders such as brittle nails [11], increase the size of collagen fibrils in Achilles tendon [12], induce fibroblast density, and enhance formation of collagen fibrils in dermis etcetera [13]. Traditionally, collagens are derived from livestock sources, such as bovine hide and bones as well as swine 
skin [14]. Because of the outbreaks of bovine spongiform encephalopathy (BSE) and foot-and-mouth disease (FMD) in recent years, collagens and collagen-derived products from such sources may have intimidated considerable users [15]. Collagens and collagen-derived products from swine sources have an additional disadvantage as religious sake in some parts of the world [16]. "Fish wastes" therefore turn out to be a good alternative. In fish processing, a large portion of wastes would generate, which typically accounts for $50 \%-$ $70 \%$ of raw materials including skins, bones, scales, viscera, and heads [17]. These so-called "wastes" were difficult to handle, generally used as low-value feedstuffs or fertilizers as a whole, so that serious environmental issues arise from time to time [18]. Given $30 \%$ or so of collagen contained in these "wastes", they have drawn great attention lately [19].

The stratum corneum (SC) has been known to minimize passive water loss from the body, to ward off absorption of chemicals in the environment, and to prevent microbial invasion $[19,20]$. Topical administration of cosmetics or biomedical materials on skin therefore has to overcome the stratum corneum barrier so as to reach the fibroblast cells in the dermal layer. Raw collagens derived from whatever sources without any process for either cosmetic or biomedical purposes may not have desirable effects, for instance the type I collagen proliferation. We considered factors such as molecular volume (MV) or molecular weight (MW) of collagens may play a key role on their penetration ability when administrated topically [21], of which relevant reports remained few.

In this paper, we prepared testing collagens using scale wastes of tilapia (Oreochromis sp), which is a high valueadded fish in Taiwan fishery. The prepared collagens formulated into skin essence were assessed for their physiological effects on facial skin in users. In addition, we determined the levels of fibroblasts proliferation and collagen synthesis in embryonic fibroblast cell lines after being subjected to FSCP treatments. Given molecular sizes of FSCPs on transdermal efficiencies were also explored. In fact, our results showed that the transdermal penetration efficiency of FSCPs is positively correlated to given FSCPs' molecular sizes and structural features in a nude mice model.

\section{Materials and Methods}

2.1. Preparation of Fish Scale Collagen Peptides (FSCPs). Fish scale collagen peptides (FSCPs) were isolated from tilapia (Oreochromis sp.) scales (Ko-Fwu Fishes Co., Taiwan) by enzymatic hydrolysis according to our patented protocol [22]. In short, fish scales were washed to rid of impurities and then heated for 15 minutes at $121^{\circ} \mathrm{C}$ to soften scales. The heated scales $(200 \mathrm{~g})$ were smashed into small pieces by disperser (Kinematica, NY, USA), then subjected to hydrolysis under $1 \%$ Protease $\mathrm{N}$ for 2.5 hours and $0.5 \%$ Flavourzyme (Novozymes, Chiba-shi, Japan) for another 0.5 hour at an optimal $\mathrm{pH}$ and temperature. Hydrolysates were stirred and heated in a boiling water bath for $10 \mathrm{~min}$ to inactivate enzymes. Then, the hydrolysates were centrifuged at $12,000 \mathrm{~g}$ for $20 \mathrm{~min}$. The supernatants (FSCPs) were lyophilized and stored at $-20^{\circ} \mathrm{C}$ for use.
2.2. Moisture Contents and Relative Elasticity Assays. FSCPbased skin essences were formulated into 5\%,7\%, and $10 \%$ of total FSCPs as the common basal ingredients. Sixty-two voluntary Taiwanese women (within 23 to 60 years of age) were subjected to the FSCP-based skin essence treatment on facial skin twice a day for 30 days. These test subjects were forbidden using any other cosmetic products during the test. Moisture contents and relatively elasticity of facial skins were measured every other week (at the $0,2 \mathrm{nd}$, and 4 th week) by the skin probe of Cutometer MAP 580 (KOKO, Leichlingen, Germany) at $20-22^{\circ} \mathrm{C}$ and at $40 \%-60 \%$ relative humidity. Each measurement took place at $30 \mathrm{~min}$ after washing face with DI water.

2.3. Cell Proliferation and Collagen Release Assays. About 2 $\times 10^{5}$ Detroit 551 cells (human embryonic skin fibroblast cell line, ATCC CCL-110) and STO cells (mouse embryonic fibroblast cell line, ATCC CRL-1503) were seeded into each well of a 96-well plate and maintained in eagle's minimum essential medium (EMEM) and Dulbecco's modified Eagle's medium (Sigma, St. Louis, MO, USA) plus 10\% fetal bovine serum (FBS) (Invitrogen, San Diego, CA, USA), respectively. Cells were cultured overnight. The medium was refreshed with new medium containing $0,0.4,0.8,1.6,3.2,6.3,12.5$, $25,50,100$, and $200 \mathrm{mM}$ FSCPs; cells were incubated for 48 hours before MTT and procollagen type I production assays. For MTT assay, $100 \mu \mathrm{l}$ of the MTT solution [a mixture of 3-(4,5-dimethylthiazol-2-yl)-2,5-diphenyl tetrazolium bromide (MTT) and yolk lipoprotein (YLP) (Invitrogen, San Diego, CA, USA) in PBS] was added into wells and reacted for 3 hours at $37^{\circ} \mathrm{C}$. The plates were stirred for $2 \mathrm{~min}$, and corresponding absorbance was recorded at $570 \mathrm{~nm}$ using an ELISA reader [6]. For the procollagen type I production assay, the procollagen type I C-peptide ELISA kit (Takara Bio Inc, Otsu, Japan) was used. Experimental procedures followed the manufacturer's instruction. In short, $20 \mu \mathrm{l}$ of culture medium and $100 \mu \mathrm{l}$ of the antibody-POD conjugate solution were sequentially added into microtiter plates and reacted for 3 hours at $37^{\circ} \mathrm{C}$. After $4 \times 5 \mathrm{~min}$ washing with the provided washing buffer solution $(400 \mu \mathrm{l} /$ well $), 100 \mu \mathrm{l}$ of the substrate solution was added in. After 15 min room temperature incubation, the stop solution $(100 \mu \mathrm{l})$ was added in; corresponding absorbance was recorded at $450 \mathrm{~nm}$ using an ELISA reader.

2.4. Molecular Size Effects of FSCPs. The enzymatic hydrolysates from the fish scales were fractionationed into five different peptide pools, using the Millipore minitan system (Millipore, Bedford, MA) with four different molecular weight cutoff membranes $(5,3,1$, and $0.5 \mathrm{kDa})$. A $50 \mu \mathrm{l}$ aliquot of fish scale hydrolysate was loaded onto a Superdex peptide $10 / 300 \mathrm{GL}(10 \mathrm{~mm} \times 300 \mathrm{~mm} \times 13 \mu \mathrm{m})$ gel filtration column (Pharmacia, Uppsala, Sweden) and eluted with $200 \mathrm{mM}$ phosphate buffer (with $0.25 \mathrm{M} \mathrm{NaCl}$, $\mathrm{pH} 7.8$ ) at a flow rate of $0.5 \mathrm{~mL} / \mathrm{min}$. FSCPs were calibrated against protein standards (Bio-Rad, California, USA) of cytochrome $\mathrm{C}(\mathrm{Mr}=12500 \mathrm{Da})$, aprotinin $(\mathrm{Mr}=6512 \mathrm{Da})$, vitamin $\mathrm{B} 12(\mathrm{Mr}=1355 \mathrm{Da})$, and cytidine $(\mathrm{Mr}=246 \mathrm{Da})$. 
Average molecular weights (MW) of fractionationed FSCPs were determined to be $4500,3500,2000$, and $1300 \mathrm{Da}$, respectively; each fraction was lyophilized for use.

2.5. FSCPs Labeled with Fluorescein Isothiocyanate. FITClabeled FSCPs were obtained by using the Fluoro Tag FITC conjugation kit (Sigma, St. Louis, MO, USA). The FITClabeled FSCPs were prepared based on a conjugation reaction between the isothiocyanate group in FITC and the primary amino groups in collagen peptides [23]. In short, FSCPs solution $(750 \mathrm{ppm}, 1 \mathrm{~mL})$ was rebuffered with a phosphate buffer solution ( $\mathrm{pH}$ 7.4). The new FSCPs solution was mixed with $0.25 \mathrm{~mL}$ of $0.1 \mathrm{M}$ sodium carbonate-bicarbonate buffer ( $\mathrm{pH}$ 9.0) containing FITC, which was gently stirred for 2 hours at room temperature. The FSCPs labeled with FITC were isolated by Sephadex G-25 column (Bio-Rad, California, USA), where phosphate saline $(10 \mathrm{mM}$ sodium phosphate, $27 \mathrm{mM} \mathrm{KCl}, 138 \mathrm{mM} \mathrm{NaCl}$, and $\mathrm{pH} 7.4$ ) was used as the mobile phase.

2.6. In Vivo Transdermal Delivery Efficiency of FITC-Labeled FSCPs. Six-week-old female $\mathrm{C} 3 \mathrm{H} / \mathrm{HeN}$ mice were anesthetized using acepromazine maleate (i.p.). Hairs covering the areas of abdomen skin were removed with a shaver. Residual hairs were removed by hair-remove-cream (Yanagiya, Japan). Mice were subjected to destratum corneum by treating mice with $10 \%$ alpha hydroxyl acids (AHAs; BIOPEUTIC, USA) for 5 minutes and cleanup for use. An aliquot of $25 \mu \mathrm{g}$ FITC-labeled FSCPs in sterile DI water $(100 \mu \mathrm{l})$ was introduced into nonwoven fabrics (cosmetics mask; Widetex Biotech Co., Taiwan). The fabric covered $1 \mathrm{~cm}^{2}$ of hairless dorsal back skin which then was topped with $1.5 \mathrm{~cm}^{2}$ transparent dressing film (Tegaderm, Neuss, Germany). The nonwoven fabric was removed after one hour. The skin tissues treated with the FITC-labeled FSCPs were embedded in O.C.T. Embedding Medium (Sakura Finetek USA, Torrance, California) and sectioned into $10 \mu \mathrm{m}$ thickness. The skin tissues were observed under fluorescence microscopy (BX-51, Olympus, Japan) equipped with a digital camera [24].

2.7. The Franz-Type Diffusion Cell Model. The procedure was modified from the method described by Kim et al. [25]. Nude mice skin was mounted on the receptor compartment of the Franz-type diffusion cell (PermeGear, HT, USA). A phosphate buffer solution $(6.80 \mathrm{~g} / \mathrm{l}$ potassium phosphate monobasic, $3.78 \mathrm{~g} / \mathrm{l}$ sodium chloride, and $0.235 \mathrm{~g} / 1$ sodium acid; $\mathrm{pH}$ 7.4) containing $750 \mathrm{ppm}$ a given fraction of FSCPs was added in the tight interface, facing nude mice skin. The donor cap was filmed with a parafilm. An isotonic phosphate buffer solution ( $\mathrm{pH}$ 7.4) was used as the receptor solution. The receptor solution was stirred by a magnetic follower rotating at $250 \mathrm{rpm}$ (which would increase mixing efficiency and reduce tendency of forming a stagnant boundary layer next to membrane surface). Samples were performed at 1, 2, $3,4,6$, and 24 hours. The amount of total collagen peptides from the receptor solution was measured by the BCA protein assay (Pierce, Rockford, USA).
The cumulative penetration amount $(\mu \mathrm{g})=[C(\mathrm{ppm}) \times$ $\left.V_{i}(\mathrm{~mL})\right]+\sum_{n-1}\left(C \times V_{s}\right)$

$\left(C=\right.$ concentration; $V_{i}=$ initial volume; $V_{s}=$ sampling volume).

The unit area cumulative penetration amount $\left(\mu \mathrm{g} / \mathrm{cm}^{2}\right)=$ cumulative penetration amount/the measure of area $\left(0.66 \mathrm{~cm}^{2}\right)$.

\subsection{Transdermal Penetration Efficiency of FITC-Labeled} FSCPs Determined by Confocal Spectral Microscope. Various sizes of FSCPs were labeled with FITC, which would penetrate into nude mice skins in the Franz-type diffusion cell model. The skins of nude mice treated with FITC alone served as a control. After one hour, the treated skin tissues were dissected out, embedded in O.C.T. compound and sectioned in $10 \mu \mathrm{m}$ thickness. The slides were then mounted on and treated with a blocking solution (95\% ethanol and $5 \%$ acetic acid). After rinsed with PBS for 20 seconds and then rinsed with $50 \%, 70 \%, 90 \%$, and $100 \%$ ethanol for 30 seconds, the slides were observed under a confocal spectral microscope (TCS SP5, Leica, Wetzlar, Germany).

2.9. Statistical Analysis. The graphs and statistical analyses were performed using SigmaPlot and SigmaStat. The statistical analyses between groups were determined by nonparametric one-way analysis of variance (Krmskal-Wallis test) and Mann-Whitney tests. Differences were considered significant if the $P$ value was $\leq .05$.

\section{Results}

3.1. Effects of FSCPs on Human Facial Skin Moisture Contents and Relative Elasticity. While collagen peptides have been well documented to be an ideal material in cosmetic industries [26], fish scale collagen peptides (FSCPs) as a sustainable collagen source however did not draw great attention. In this study, we examined the treating effects of FSCPs as a major component in a skin essence on the facial skin moisture contents and relative elasticity by using standard professional skin probes. The FSCP-based skin essences were first formulated into $5 \%, 7 \%$, and $10 \%$ of total FSCPs as the common basal ingredients. Sixty-two voluntary Taiwanese women (within 23 to 60 years of age) were subjected to the FSCP-based skin essence treatment on facial skin twice a day for 30 days. The effects with all FSCPbased skin essences on the skin moisture contents were found improving significantly but in a time- and dose-dependent manner during the testing course $(P \leq .05$; Figure 1(a)). Likewise, the effects with all FSCP-based skin essences on the relative elasticity of facial skin were also found increasing in the same manner as for the skin moisture test by $24 \%, 33 \%$, and $35 \%$, respectively $(P \leq .05$; Figure $1(\mathrm{~b}))$. Thereby, the FSCP-based skin essences did improve facial skin qualities (moisture and elasticity) in a time- and dose-dependent manner in these tested subjects.

3.2. Cell Proliferation and Collagen Release in Embryonic Skin Fibroblast Cell Lines after FSCPs Treatments. To determine 

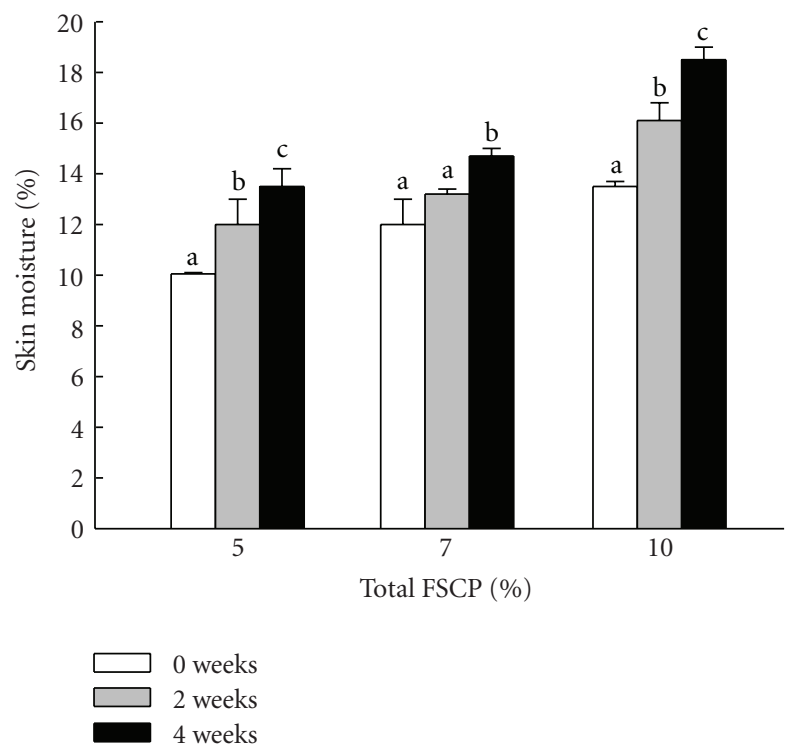

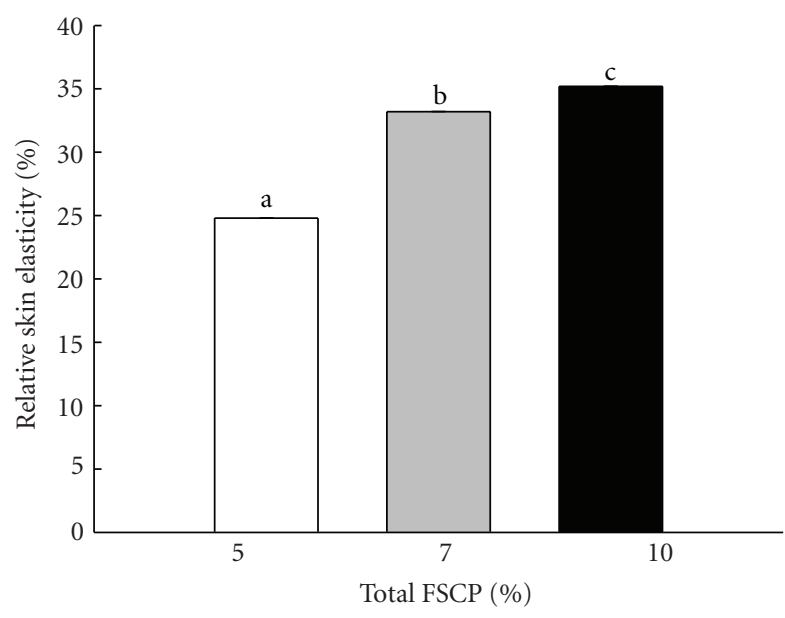

$\square \quad 0$ weeks
2 weeks
4 weeks

(a)

(b)

FIGURE 1: Effects of FSCP-based skin essence on moisture contents and relative elasticity of facial skins. The skin moisture contents (a) and relative skin elasticity (b) were measured by the professional skin measurement probes. Bars are expressed as means \pm SEM in each group for the 62 subjects. Difference letters in the same group indicate significant difference $(P \leq .05)$.

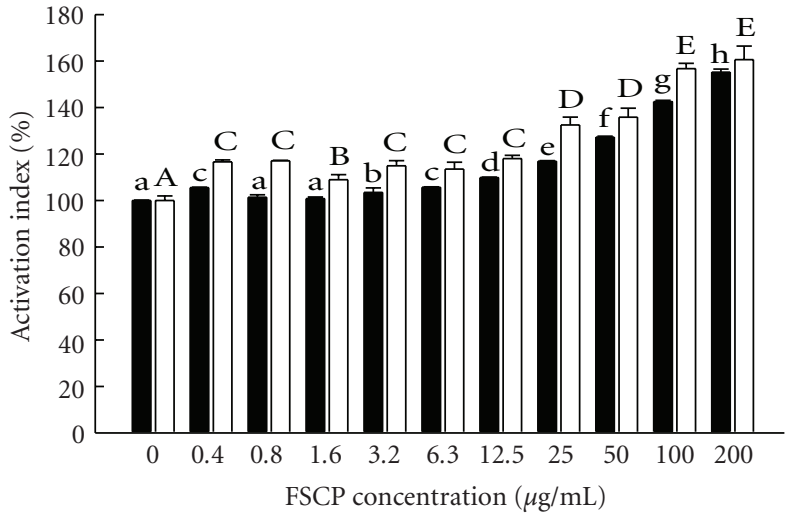
STO

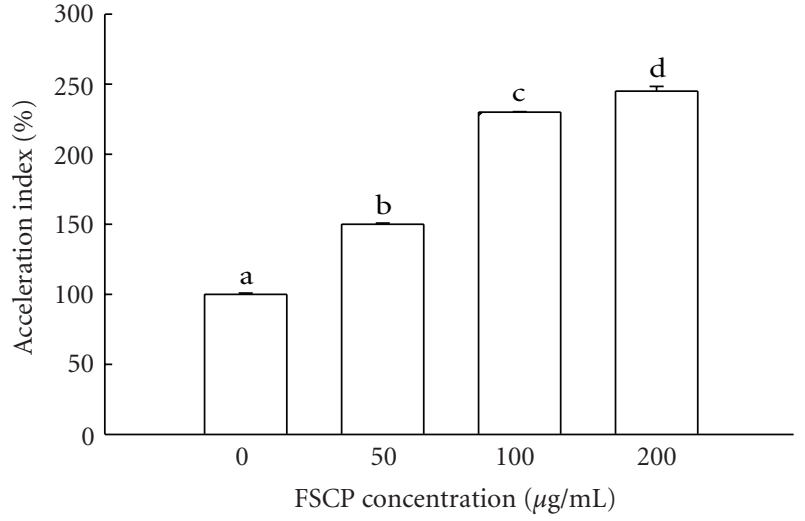

Detroit 551 STO

(a)

(b)

FIGURE 2: Cell proliferation and collagen release in embryonic skin fibroblast cell lines treated by FSCPs. Comparisons of the proliferation activity (a) and procollagen synthesis ability (b) on Detroit 551 cells (human embryonic skin fibroblast line) and STO cells (mouse embryonic fibroblast) treated with $0,0.4,0.8,1.6,3.2,6.3,12.5,25,50,100$, and $200 \mathrm{mM}$ FSCPs. The group with no FSCPs acts as a control. After 48 hours posttreatment, the samples were analyzed by MTT and procollagen type I C-peptide ELISA assays. Bars stand as means \pm standard deviation (triplicate for each group).

whether the skin quality improving was resulted from fibroblast cells proliferation and mass collagen synthesis in dermis after the FSCPs treatment, model cells Detroit 551 (human embryonic skin fibroblast) and STO (mouse embryonic fibroblast) were chosen for the purpose stated. The cells were grown in media in the presence of various concentrations of FSCPs. On the contrary, the cells grown in media without FSCPs served as controls. Cell proliferation and procollagen release in the tested cells were determined by MTT assays and procollagen type I C-peptide ELISA assays, respectively. As shown in Figure 2(a), FSCPs $(0.4-200 \mu \mathrm{g} / \mathrm{mL})$ can dosedependently stimulate the cell proliferation in both fibroblast cells. Likewise, the procollagen type I synthesis was found mass produced in a concentration-dependent manner in the cells with FSCPs treatments (Figure 2(b)). The procollagen synthesis in the presence of $200 \mu \mathrm{g} / \mathrm{mL}$ FSCPs was found most prominent and peaked as high as $250 \%$. FSCPs thereby were determined to be able to effectively stimulate and 


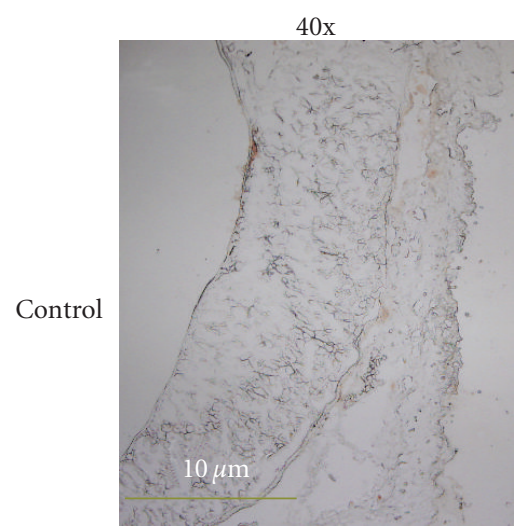

(a)

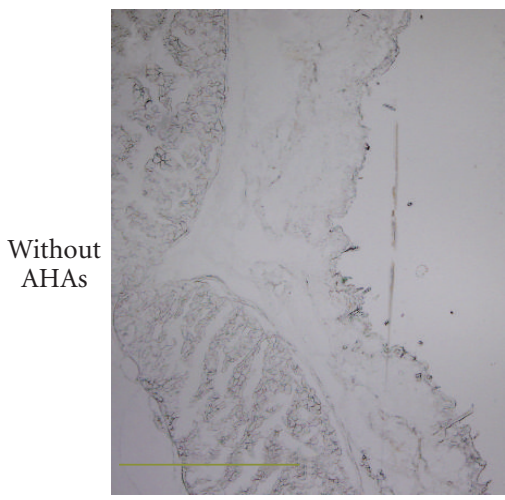

(d)

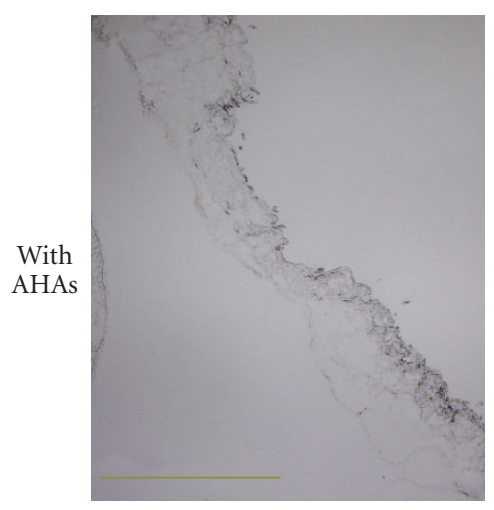

(g)

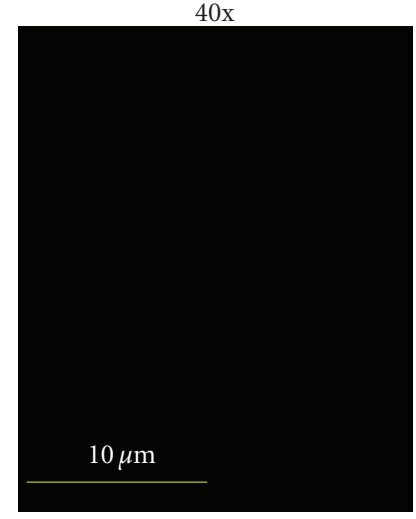

(b)

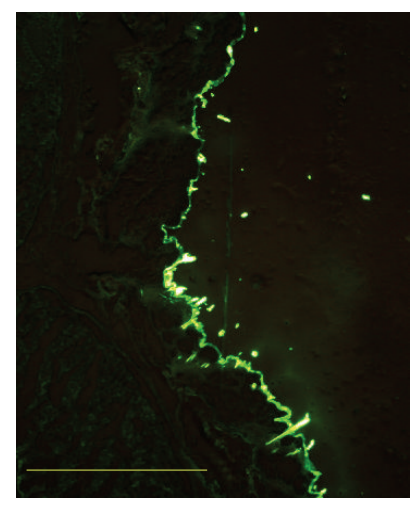

(e)

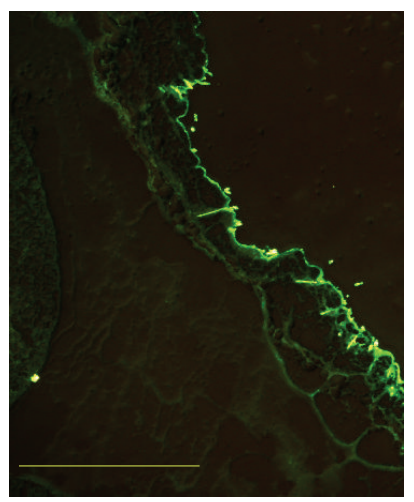

(h)

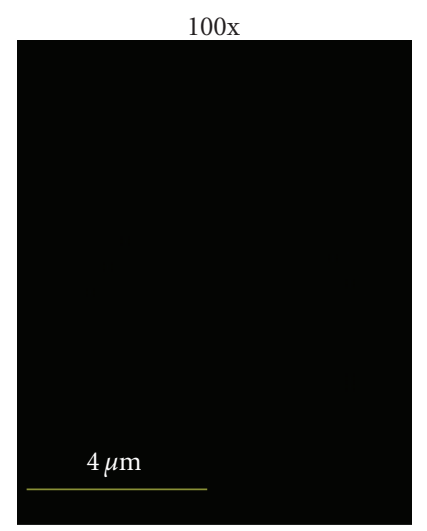

(c)

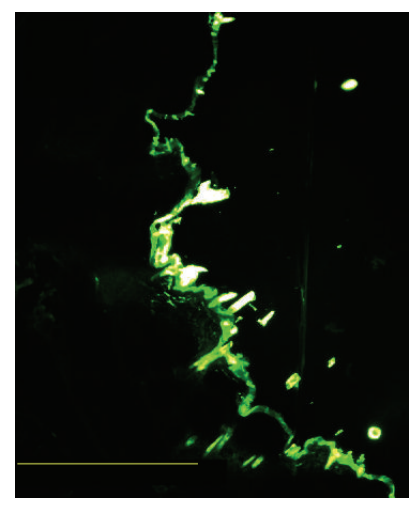

(f)

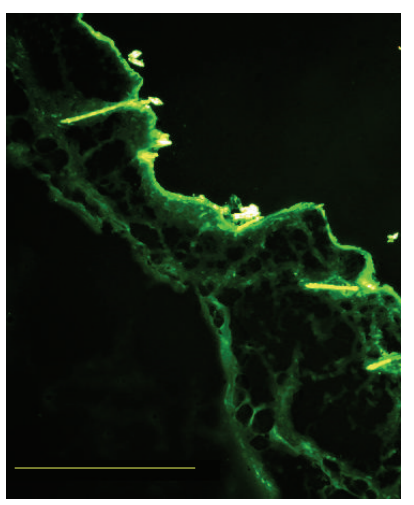

(i)

Figure 3: Fluorescence microscopic images of FITC-labeled FSCPs treated C3H/HeN mice skins. The green fluorescence is found in the follicular and epidermal areas of the vertical cross-sections of the treated skins. (a), (d), and (g) are the phase contrasts of the treated skins; (d)-(f) are groups with FITC-labeled FSCPs but without AHA pretreatment; (g)-(i) are groups with FITC-labeled FSCPs and with AHA pretreatment. (40x, 40-fold magnification; 100x, 100-fold magnification).

induce fibroblast cell proliferation and collagen synthesis so as to improve the facial skin quality.

\subsection{In Vivo Determination of Transdermal Delivery Effi-} ciency Using FITC-Labeled FSCPs. As mentioned above, the stratum corneum is the outmost layer of the epidermis, which serves as a physical barrier, for instance, preventing pathogens from invasion and also limiting desired macromolecules to pass by. To evaluate whether the processed
FSCPs are capable of overcoming the stratum corneum barrier, an animal model experiment was set to determine the transdermal efficiency of the prepared FSCPs. Six-weekold female $\mathrm{C} 3 \mathrm{H} / \mathrm{HeN}$ mice were first shaved and treated with hair-removal cream to remove residual hairs. The mice were then subjected to treatments with or without alpha hydroxyl acids (AHAs, for weakening the stratum corneum) before topical application with the nonwoven fabric that contained $25 \mu \mathrm{g}$ FITC-labeled FSCPs. The mice pretreated with $10 \%$ 


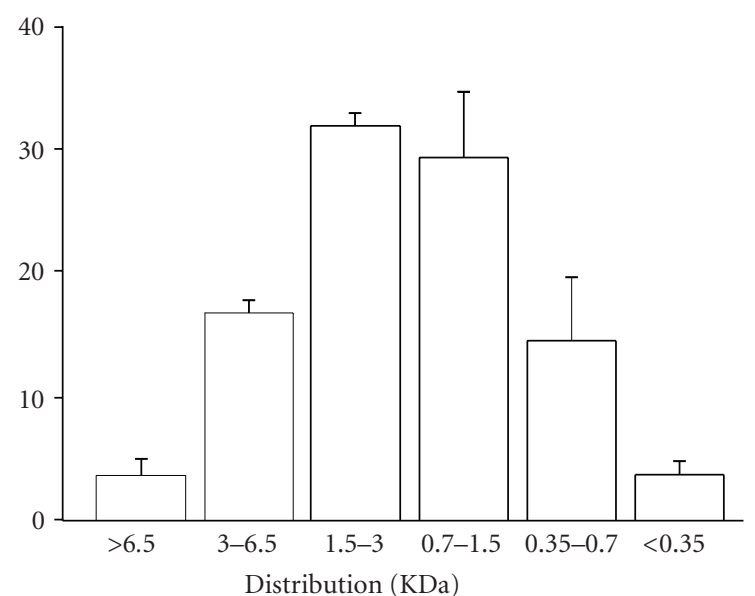

istribution $(\mathrm{KDa})$

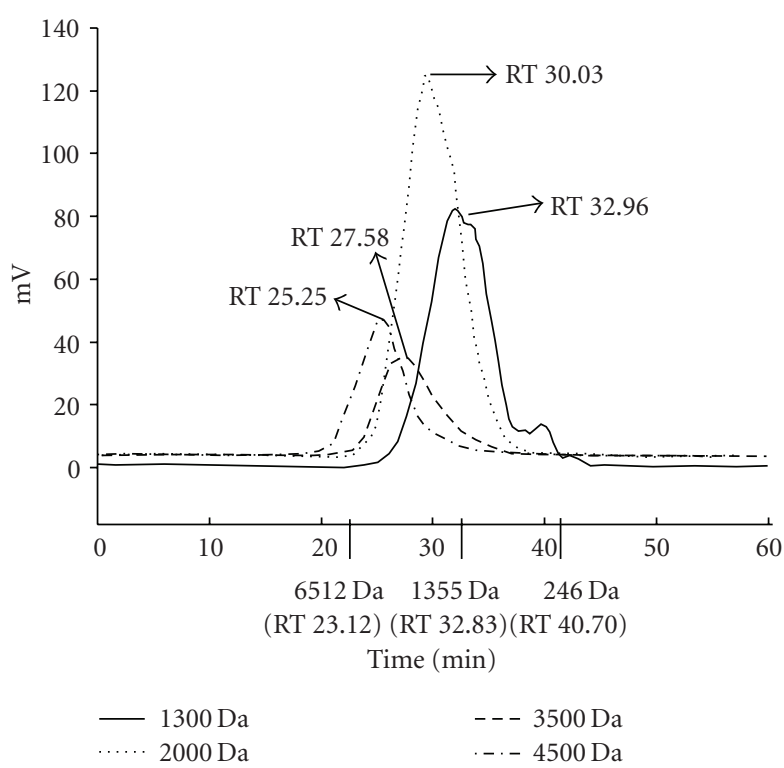

(b)

FIGURE 4: Size distribution of FSCPs. (a) Fish scale hydrolysates were separated by gel chromatography (superdex peptide 10/300 GL columns); molecular sizes were determined for each fraction; size distributions were plotted accordingly. The size distribution (KDa) is expressed as means \pm SEM. (b) Size profiles of FSCPs. Protein standards (Bio-Rad, California, USA) are cytochrome C $(\mathrm{Mr}=12500 \mathrm{Da})$, aprotinin $(\mathrm{Mr}=6512 \mathrm{Da})$, vitamin $\mathrm{B} 12(\mathrm{Mr}=1355 \mathrm{Da})$, and cytidine $(\mathrm{Mr}=246 \mathrm{Da})$. The average molecular weights $(\mathrm{MWs})$ were determined to be $4500,3500,2000$, and $1300 \mathrm{Da}$ for the four indicated groups.

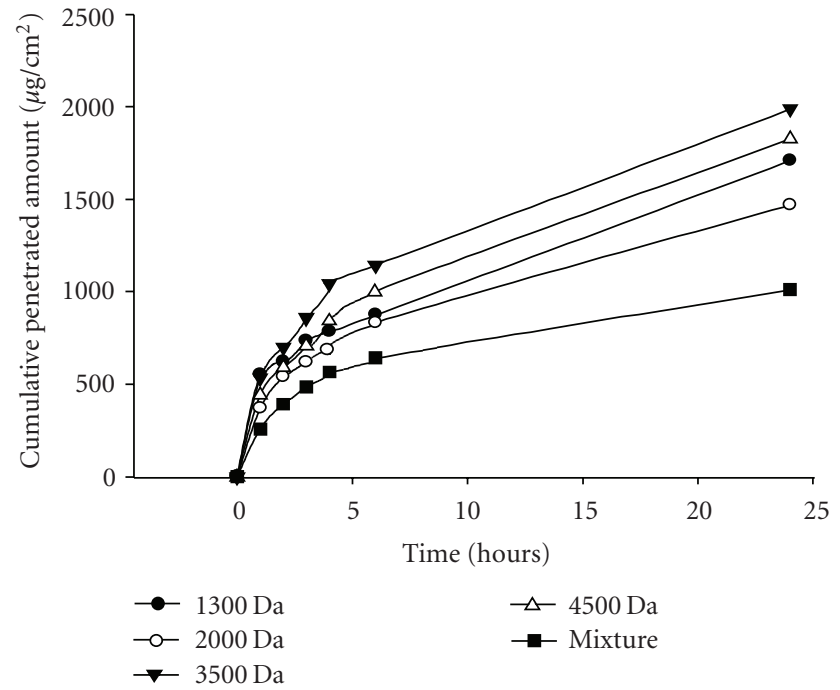

Figure 5: Transdermal permeation abilities of given FSCPs fractions. The permeation abilities of various molecular sizes of FSCPs were determined by the Franz-type diffusion cell model, performing at $1,2,3,4,6$, and 24 hours. The amounts of total collagen peptides from the receptor solutions were determined by the BCA protein assay. "Mixture" indicates the FSCP hydrolates without ultracentrifugation membrane treatment. The results shown are a representative in 3 experiments.

AHAs and topped with the nonwoven fabric containing no FITC-labeled FSCPs served as controls. These treated skins were dissected out and immediately embedded with
O.C.T. in due course. Then, the samples were sectioned, mounted on glass slides, and observed under fluorescence microscopy. As shown in Figure 3, the FITC positive signals laid mainly on the area of superficial epidermis. And, the FITC positive signals in the skin pre-treated with AHA were found deeper and brighter than those without AHA treatment. The FITC positive signals were also found in the hair follicles of epidermis. Thus, the stratum corneum barrier was determined to be a key factor that influences the penetration efficiency of FSCPs in mice skin. Nonetheless, FSCPs without AHA treatment still considerably pass by the stratum corneum, which agreed with the results in the human facial skin test.

3.4. Transdermal Penetration Ability of FSCPs in FranzType Diffusion Cell Model. We considered factors such as molecular volume (MV) or molecular weight (MW) of collagens may play a role on their penetration capability when administrated topically. The size effect for given FSCPs was determined by employing the Franz-type diffusion cell model. Fish scale hydrolates were first subjected to gel chromatography (superdex peptide 10/300 GL columns), whereby FSCP hydrolysates were fractionationed by sizes. As shown in Figure 4(a), the eluted peptides were collected into six fractions, $>6.5 \mathrm{KDa}(3.65 \%), 3-6.5 \mathrm{KDa}(16.8 \%)$, 1.5-3.0 KDa (32.0\%), 0.7-1.5 KDa (29.3\%). 0.35-0.7 KDa $(14.6 \%)$ and $<0.35 \mathrm{KDa}(3.7 \%)$. These peptide fractions were further subjected to ultrafiltration membrane refining $(5,3$, 1 , and $0.5 \mathrm{kDa}$ cutoff) into four refined groups I-IV ( $\mathrm{Mw}=$ $3-5,1-3,1-0.5$, and $<0.5 \mathrm{KDa})$ with average molecular 


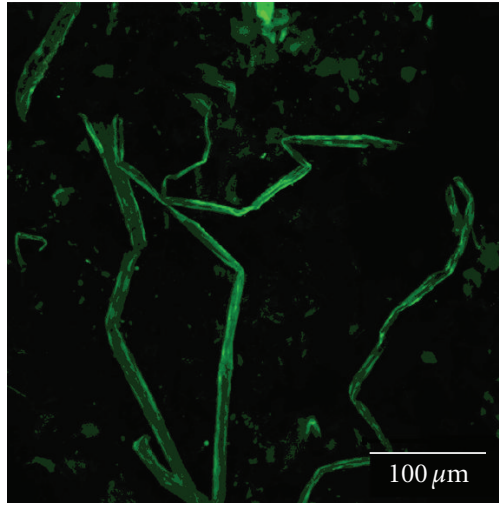

(a)

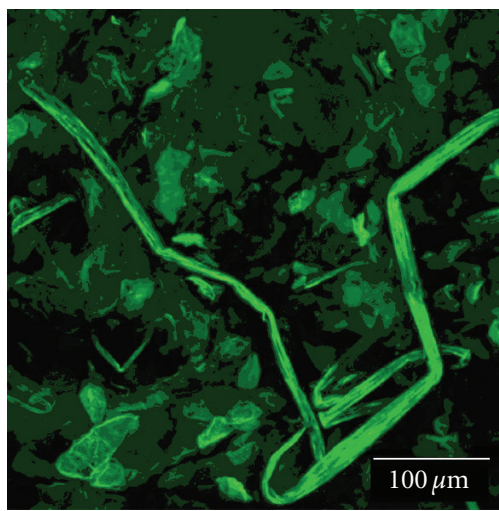

(d)

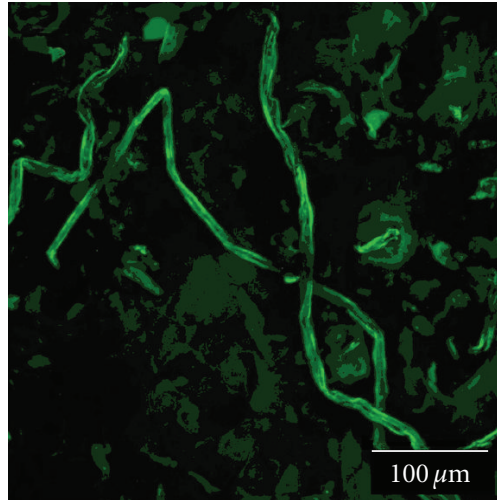

(b)

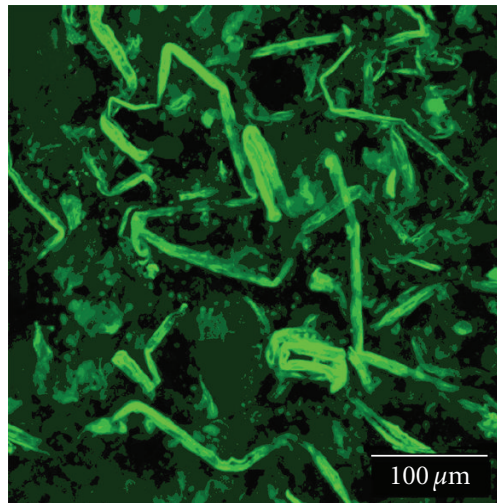

(e)

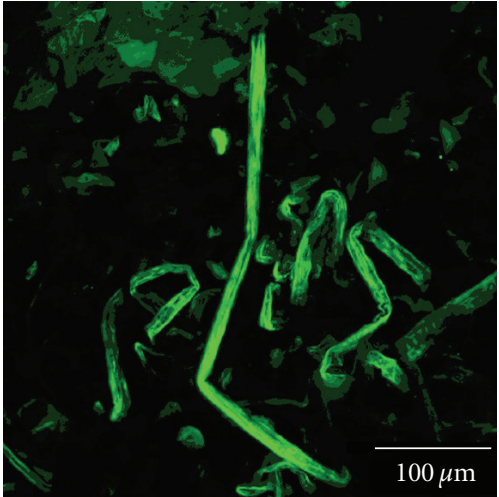

(c)

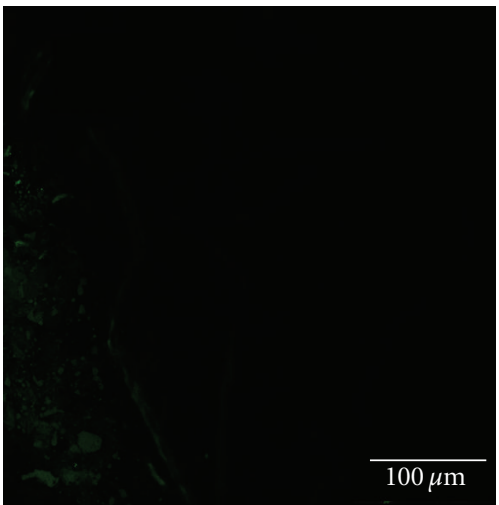

(f)

FIGURE 6: Peptide conformations of various sizes of FITC-labeled FSCPs in nude mice skins. Nude mice skins were treated with various molecular sizes of FITC-labeled FSCPs in the Franz-type diffusion cell model. The topical sites of tested nude mice skins were collected in one hour. The vertical cross-sections of the nude mice skins were imaged for FITC signals using cryosection and confocal spectral microscope systems. (a) 1,300 Da FSCPs; (b) 2,000 Da FSCPs; (c) 3,500 Da FSCPs; (d) 4,500 Da FSCPs; and (e) the mixture FSCPs (FSCPs without ultracentrifugation membrane treatment).

weights of 4500, 3500, 2000, and $1300 \mathrm{Da}$, respectively (Figure $4(\mathrm{~b})$ ). These collagen peptides were corroborated by gel chromatography (Sp-Sephadex C-25 column) against standard markers. As shown in Figure 5, all groups were found to be able to penetrate the nude mice skin in the Franztype diffusion cell model but in various extents and in a time-dependent manner. In short, groups I and II exhibited relatively better transdermal penetration ability in the first 4 hours. The highest cumulative penetration amounts for groups I and II at 24 hours were $1825.17 \pm 495.66$ and $1986.43 \pm 671.11 \mu \mathrm{g} / \mathrm{cm}^{2}$, respectively. In contrast, the FSCPs mixture (total FSCP hydrolates without ultrafiltration membrane refining) showed the lowest transdermal penetration capability $\left(1010 \pm 583.34 \mu \mathrm{g} / \mathrm{cm}^{2}\right)($ Figure 5$)$.

\subsection{Transdermal Penetration Depth and Peptide Conforma-} tion in Nude Mice Skin. To probe the penetration depth and peptide conformation for given FSCPs, each FSCPs group was first labeled with FITC and then applied to nude mice skins in the Franz-type diffusion cell model for one hour. The skin treated with given FITC-labeled FSCPs proceeded according to the same procedure described in the previous section. Interestingly, groups I (Figure 6(d)) and II (Figure 6(c)) exhibited a coil-like structure in epidermis as opposed to groups III (Figure 6(b)) and IV (Figure 6(a)) which showed less folded or in a linear form. In contrast, the mixed FSCPs did not have a defined structure (Figure 6(e)). The penetration depths for each FSCPs group were also measured by confocal spectral microscopy. The distances for groups IV-I and the mixture were 21.21, 21.44, 28.72, 27.73, and $14.58 \mu \mathrm{m}$, respectively. The heavier FSCPs penetrated deeper than the lighter. As a result, the transdermal penetration efficiency of given FSCPs are positively correlated to FSCPs' sizes and/or conformations thereof. The folded structure seemed to help penetration, which however is an interesting issue for future study.

\section{Discussion}

Many reports have pointed out collagen's versatile applications, prominently for cosmetic industry [13]. Fish scale collagens appear to be an ideal collagen source as they are cheap, rich and sustainable $[25,26]$. Since collagen is the key component in maintaining the elasticity of skin, diminishing 
collagen contents in skin would therefore result in wrinkled and flabby skins [27]. In this paper, we have demonstrated the formulated FCSP-based skin essence possesses such desirable effects as increasing skin moisture contents and relative elasticity (Figure 1). The FCSPs at $200 \mu \mathrm{g} / \mathrm{mL}$ provided the best effects by manifesting embryonic skin fibroblast cell proliferations (Detroit 551 cells and STO cells) and procollagen synthesis. These results otherwise are reinforced by the reports of Katayama et al., wherein they demonstrated pentapeptides from type I collagen promoted extracellular matrix production in fibroblasts $[28,29]$.

Since the stratum corneum acts as a physical barrier for multiple purposes in skins $[19,20]$, it does influence the penetration efficiency of the FSCP-based skin essences as they were present mainly in the surface of epidermis. However, given FITC-labeled FSCPs still effectively passed by stratum corneum to epidermis and dermis in the mice model experiment. The reports of Potts and Guy agreed with the results, in which they proposed the diffusivity of a drug in skin is correlated to given molecular features of the drug [30, 31], although Lin et al. [32] considered the effect of lipophilicity in the passive diffusion permeability is the dominant factor other than the compound's molecular weight. In addition, Potts and Guy further proposed that the higher the molecular weights the greater the lipophilicity/permeability [30]. The better effects of the FSCPs' groups at Mw 3500 and $\mathrm{Mw} 4500 \mathrm{Da}$ may possess desired sizes and effective structural features. Furthermore, collagens typically exist as a right-handed triple helix structure of Gly-X-Y helix-forming repeats in a length of $300 \mathrm{~nm}$ which corresponds to 1000 amino acids or so in fibril-forming collagens (I, II, III) [3336]. Fish scale collagens are within the scope of the type I collagen [26]. The higher penetration efficiency of FSCPs at 3500 and 4500 Da may thus be attributed to the synergistic effects of high lipophilicity (adequate size) and constructive structure features.

In conclusion, the prepared FSCPs have shown to be able to effectively penetrate stratum corneum to epidermis and dermis. FSCPs at epidermis and dermis in situ can activate fibroblasts and accelerate collagen synthesis, whereby the facial skin qualities (moisture contents and relative elasticity) were significantly improved. As a consequence, the prepared FSCPs along with the formulated skin essence should hold high commercial interests and are worthy of further in-depth study for enhancing FSCPs transdermal penetration ability to a higher level.

\section{References}

[1] T. Nagai, Y. Araki, and N. Suzuki, "Collagen of the skin of ocellate puffer fish (Takifugu rubripes)," Food Chemistry, vol. 78, no. 2, pp. 173-177, 2002.

[2] R. Mayne and R. G. Brewton, "New members of the collagen superfamily," Current Opinion in Cell Biology, vol. 5, no. 5, pp. 883-890, 1993.

[3] M. Van der Rest and R. Garrone, "Collagen family of proteins," FASEB Journal, vol. 5, no. 13, pp. 2814-2823, 1991.

[4] R. R. Wickett and M. O. Visscher, "Structure and function of the epidermal barrier," American Journal of Infection Control, vol. 34 , no. 10 , supplement 1 , pp. 98-110, 2006.
[5] K. K. H. Svoboda, D. A. Fischman, and M. K. Gordon, "Embryonic chick corneal epithelium: a model system for exploring cell-matrix interactions," Developmental Dynamics, vol. 237, no. 10, pp. 2667-2675, 2008.

[6] J. Lee, E. Jung, J. Lee, et al., "Panax ginseng induces human type I collagen synthesis through activation of Smad signaling," Journal of Ethnopharmacology, vol. 109, no. 1, pp. 29-34, 2007.

[7] J. Wu, M. Fujioka, K. Sugimoto, G. Mu, and Y. Ishimi, "Assessment of effectiveness of oral administration of collagen peptide on bone metabolism in growing and mature rats," Journal of Bone and Mineral Metabolism, vol. 22, no. 6, pp. 547-553, 2004.

[8] Y.-I. Koyama, A. Hirota, H. Mori, et al., "Ingestion of gelatin has differential effect on bone mineral density and body weight in protein undernutrition," Journal of Nutritional Science and Vitaminology, vol. 47, no. 1, pp. 84-86, 2001.

[9] R. W. Moskowitz, "Role of collagen hydrolysate in bone and joint disease," Seminars in Arthritis and Rheumatism, vol. 30, no. 2, pp. 87-99, 2000.

[10] J. Scala, N. R. S. Hollies, and K. P. Sucher, "Effect of daily gelatine ingestion on human scalp hair," Nutrition Reports International, vol. 13, no. 6, pp. 579-592, 1976.

[11] T. L. Tyson, "The effect of gelatin on fragile fingernails," Journal of Investigative Dermatology, vol. 14, pp. 323-325, 1950.

[12] J. Minaguchi, Y.-I. Koyama, N. Meguri, et al., "Effects of ingestion of collagen peptide on collagen fibrils and glycosaminoglycans in Achilles tendon," Journal of Nutritional Science and Vitaminology, vol. 51, no. 3, pp. 169-174, 2005.

[13] N. Matsuda, Y.-I. Koyama, Y. Hosaka, et al., "Effects of ingestion of collagen peptide on collagen fibrils and glycosaminoglycans in the dermis," Journal of Nutritional Science and Vitaminology, vol. 52, no. 3, pp. 211-215, 2006.

[14] M. Ogawa, R. J. Portier, M. W. Moody, J. Bell, M. A. Schexnayder, and J. N. Losso, "Biochemical properties of bone and scale collagens isolated from the subtropical fish black drum (Pogonia cromis) and sheepshead seabream (Archosargus probatocephalus)," Food Chemistry, vol. 88, no. 4, pp. 495-501, 2004.

[15] A. Jongjareonrak, S. Benjakul, W. Visessanguan, T. Nagai, and M. Tanaka, "Isolation and characterisation of acid and pepsinsolubilised collagens from the skin of Brownstripe red snapper (Lutjanus vitta)," Food Chemistry, vol. 93, no. 3, pp. 475-484, 2005.

[16] M. Sadowska, I. Kołodziejska, and C. Niecikowska, "Isolation of collagen from the skins of Baltic cod (Gadus morhua)," Food Chemistry, vol. 81, no. 2, pp. 257-262, 2003.

[17] P. Kittiphattanabawon, S. Benjakul, W. Visessanguan, T. Nagai, and M. Tanaka, "Characterisation of acid-soluble collagen from skin and bone of bigeye snapper (Priacanthus tayenus)," Food Chemistry, vol. 89, no. 3, pp. 363-372, 2005.

[18] K. Gelse, E. Pöschl, and T. Aigner, "Collagens-structure, function, and biosynthesis," Advanced Drug Delivery Reviews, vol. 55, no. 12, pp. 1531-1546, 2003.

[19] P. M. Elias and E. H. Choi, "Interactions among stratum corneum defensive functions," Experimental Dermatology, vol. 14, no. 10, pp. 719-726, 2005.

[20] P. M. Elias, "Stratum corneum defensive functions: an integrated view," Journal of Investigative Dermatology, vol. 125, no. 2, pp. 183-200, 2005.

[21] B. Baert, E. Deconinck, M. V. Gele, et al., "Transdermal penetration behaviour of drugs: CART-clustering, QSPR and 
selection of model compounds," Bioorganic and Medicinal Chemistry, vol. 15, no. 22, pp. 6943-6955, 2007.

[22] C. H. Wu and H. J. Chai, "Collagen of fish scale and method of macking thereof," Taiwan Invention patent publication number I 263678, 2006.

[23] Z. Ma and L.-Y. Lim, "Uptake of chitosan and associated insulin in Caco-2 cell monolayers: a comparison between chitosan molecules and chitosan nanoparticles," Pharmaceutical Research, vol. 20, no. 11, pp. 1812-1819, 2003.

[24] T. D. Russell, A. Fischer, N. E. Beeman, E. F. Freed, M. C. Neville, and J. Schaack, "Transduction of the mammary epithelium with adenovirus vectors in vivo," Journal of Virology, vol. 77, no. 10, pp. 5801-5809, 2003.

[25] N. Kim, A. F. El-Kattan, C. S. Asbill, et al., "Evaluation of derivatives of 3-(2-oxo-1-pyrrolidine) hexahydro- $1 H$ azepine-2-one as dermal penetration enhancers: side chain length variation and molecular modeling," Journal of Controlled Release, vol. 73, no. 2-3, pp. 183-196, 2001.

[26] L. Wang, X. An, F. Yang, Z. Xin, L. Zhao, and Q. Hu, "Isolation and characterisation of collagens from the skin, scale and bone of deep-sea redfish (Sebastes mentella)," Food Chemistry, vol. 108, no. 2, pp. 616-623, 2008.

[27] H. Tanaka and S. Hasegawa, "Skin permeable collagen peptide preventing wrinkle formation induced by photoaging," Biotechnology Industrial, vol. 22, no. 9, pp. 18-23, 2005.

[28] M. W. Kofford, L. B. Schwartz, N. M. Schechter, D. R. Yager, R. F. Diegelmann, and M. F. Graham, "Cleavage of type I procollagen by human mast cell chymase initiates collagen fibril formation and generates a unique carboxyl-terminal propeptid," Journal of Biological Chemistry, vol. 272, no. 11, pp. 7127-7131, 1997.

[29] K. Katayama, J. Armendariz-Borunda, R. Raghow, A. H. Kang, and J. M. Seyer, "A pentapeptide from type I procollagen promotes extracellular matrix production," Journal of Biological Chemistry, vol. 268, no. 14, pp. 9941-9944, 1993.

[30] R. O. Potts and R. H. Guy, "Predicting skin permeability," Pharmaceutical Research, vol. 9, no. 5, pp. 663-669, 1992.

[31] R. H. Guy and R. O. Potts, "Structure-permeability relationships in percutaneous penetration," Journal of Pharmaceutical Sciences, vol. 81, no. 6, pp. 603-604, 1992.

[32] R.-Y. Lin, C.-W. Hsu, and W.-Y. Chen, "A method to predict the transdermal permeability of amino acids and dipeptides through porcine skin," Journal of Controlled Release, vol. 38, no. 2-3, pp. 229-234, 1996.

[33] K. Kuhn, "The collagen family-variations in the molecular and supermolecular structure," Rheumatology, vol. 10, pp. 29-69, 1986.

[34] K. A. Piez, "Molecular and aggregate structure of the collagens," in Extracellular Matrix Biology, K. A. Pietz and H. Reddi, Eds., pp. 1-39, Elsevier, New York, NY, USA, 1984.

[35] J. F. Bateman, S. R. Lamande, and J. A. M. Ramshaw, "Collagen superfamily," in Extracellular Matrix, W. D. Comper, Ed., pp. 22-67, Harwood, Melbourne, Australia, 1996.

[36] K. Von der Mark, "Structure, biosynthesis and gene regulation of collagens in cartilage and bone," in Dynamics of Bone and Cartilage Metabolism, M. J. Seibel, S. P. Robins, and J. P. Bilezikian, Eds., pp. 3-29, Elsevier, New York, NY, USA, 1999. 

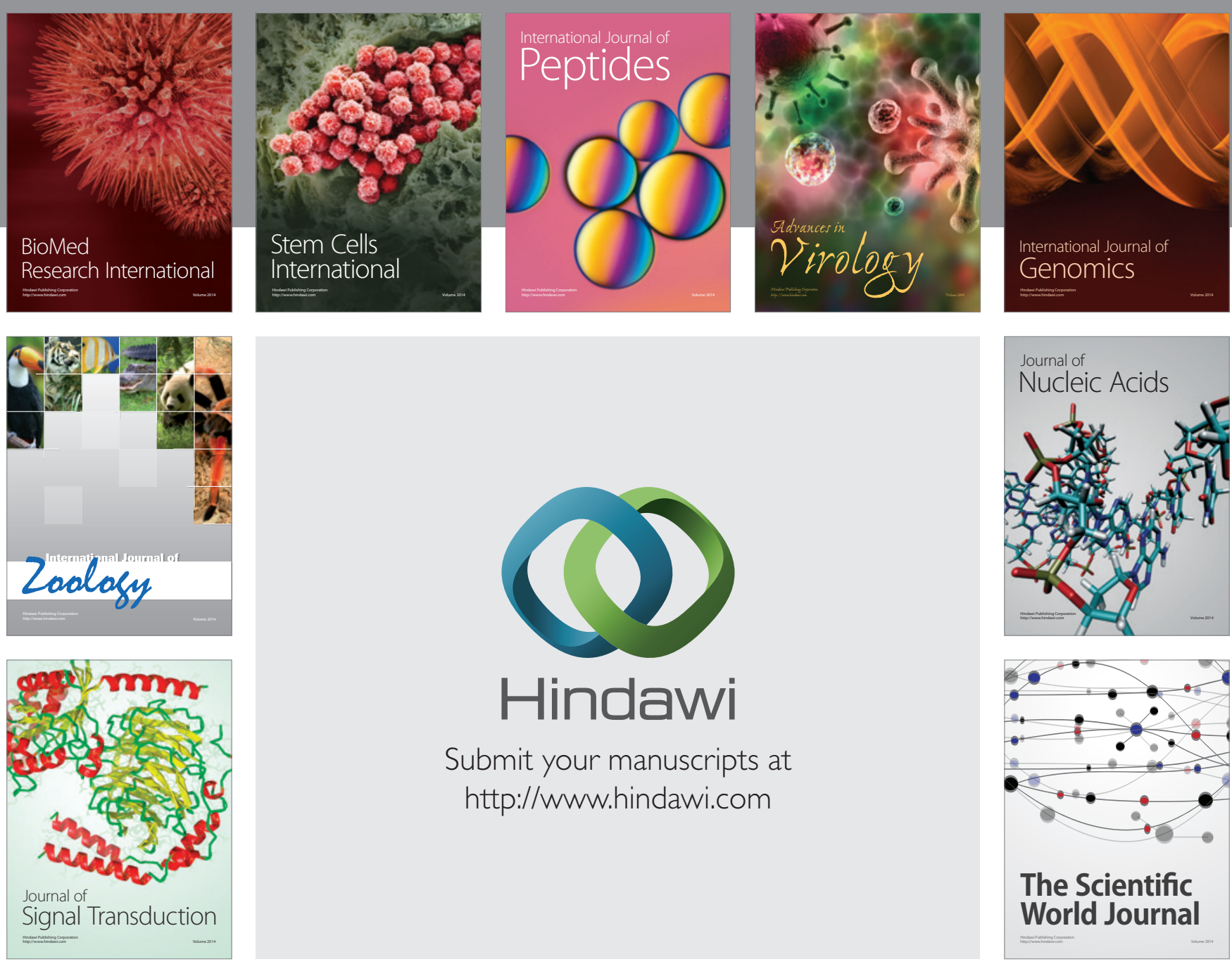

Submit your manuscripts at

http://www.hindawi.com
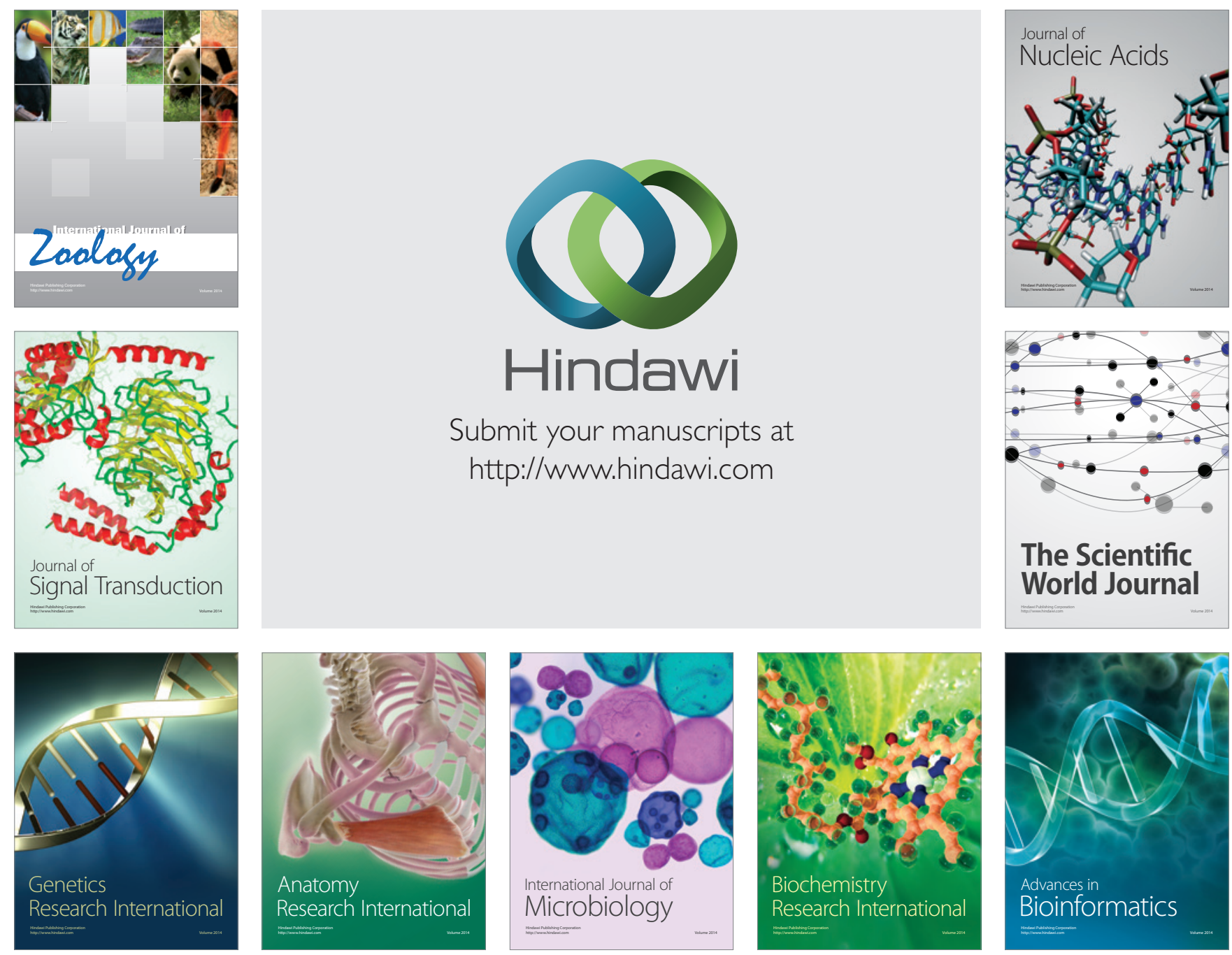

The Scientific World Journal
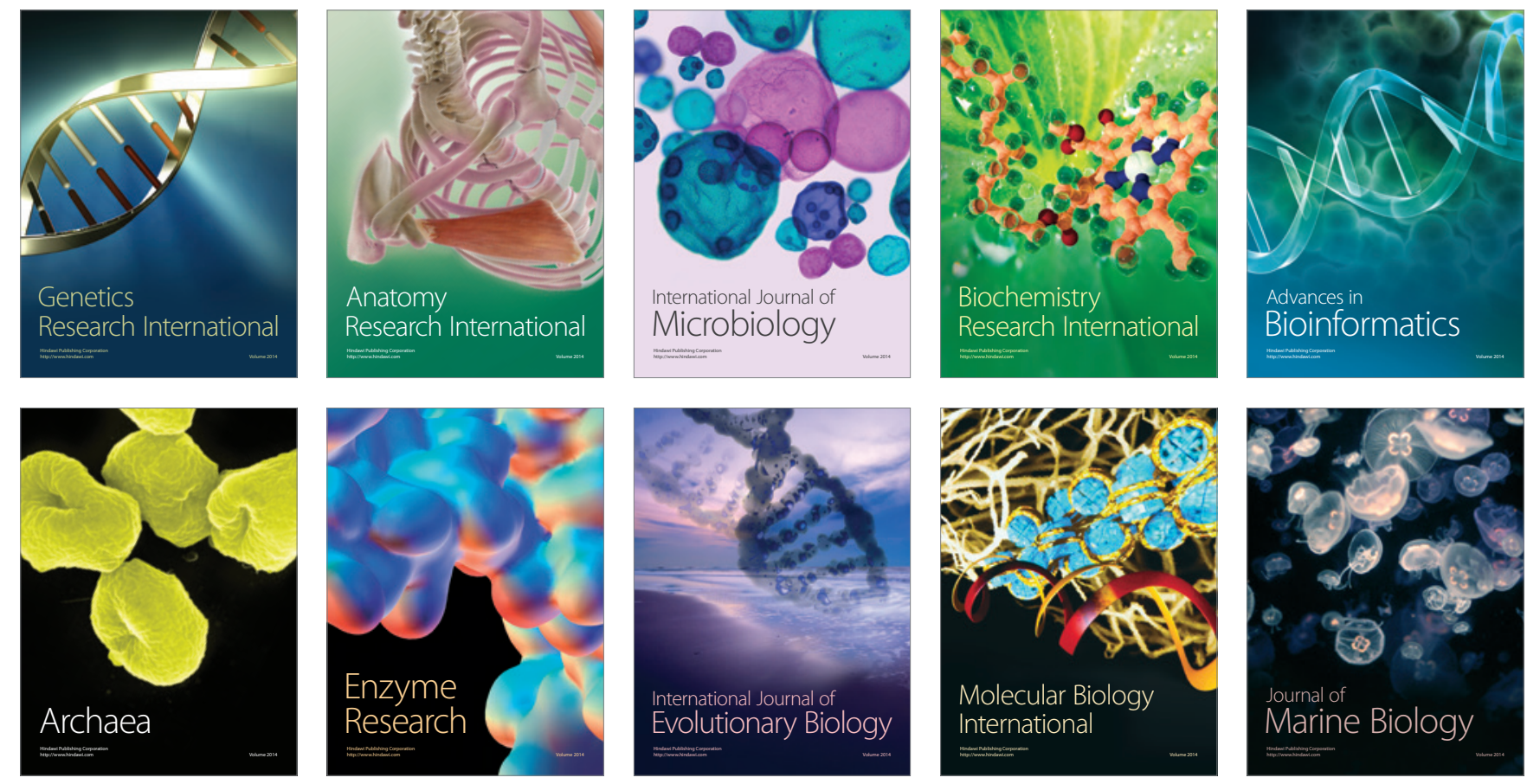